\title{
Fast Diffusion Flow on Manifolds of Nonpositive Curvature
}

\author{
Matteo Bonforte ${ }^{1,3,4}$, Gabriele Grillo ${ }^{2,5}$, Juan Luis Vazquez ${ }^{1,6}$
}

July 14, 2006

\begin{abstract}
We consider the fast diffusion equation (FDE) $u_{t}=\Delta u^{m}(0<m<1)$ on a nonparabolic Riemannian manifold $M$. Existence of weak solutions holds. Then we show that the validity of Euclidean-type Sobolev inequalities implies that certain $\mathrm{L}^{p}-\mathrm{L}^{q}$ smoothing effects of the type $\|u(t)\|_{q} \leq C t^{-\alpha}\left\|u_{0}\right\|_{p}^{\gamma}$, the case $q=\infty$ being included. The converse holds if $m$ is sufficiently close to one. We then consider the case in which the manifold has the addition gap property $\min \sigma(-\Delta)>0$. In that case solutions vanish in finite time, and we estimate from below and from above the extinction time.
\end{abstract}

Keywords. Nonlinear evolutions, singular parabolic equations, fast diffusion, Riemannian manifolds, asymptotics.

Mathematics Subject Classification. 35B45, 35B65, 35K55, 35K65.

(1) Departamento de Matemáticas, Universidad Autónoma de Madrid, Campus de Cantoblanco, 28049 Madrid, Spain

(2) Dipartimento di Matematica, Politecnico di Torino, corso Duca degli Abruzzi 24, 10129 Torino, Italy

(3) Ceremade, Université Paris Dauphine, Place de Lattre de Tassigny, F-75775 Paris Cédex 16, France

(4) e-mail address: bonforte@calvino.polito.it

(5) e-mail address: gabriele.grillo@polito.it

(6) e-mail address: juanluis.vazquez@uam.es 


\section{Introduction}

This paper is a further contribution to the study of nonlinear diffusion on manifolds. We consider a complete Riemannian manifold $M$ of dimension $d \geq 3$, having infinite volume, and study the existence and behaviour of the solutions $u(x, t)$ of the fast diffusion equation (FDE)

$$
\partial_{t} u=\triangle\left(u^{m}\right), \quad x \in M, t>0,
$$

where $\Delta$ is the Laplace-Beltrami operator on $M$, the exponent $m \in(0,1)$, and we use the standard convention $u^{m}:=u|u|^{m-1}$ for solutions that change sign. We cover three main issues: existence, regularizing properties (also called smoothing effects) and extinction in finite time. Convenient assumptions on the type of manifold are needed in the different stages of the work.

Our first task is to guarantee the existence of weak solutions. We assume that $M$ is a nonparabolic Riemannian Manifold (see below). The existence technique is based on an abstract result of Brezis [8] on semigroups generated by a maximal monotone operator in a Hilbert space. The space turns out to be $H^{-1}(M)$. In dealing with manifolds we follow the main ideas explained in Chapter 10 of the book [17] in the compact case. We show that the method works also in the setting of nonparabolic manifolds and derive the basic properties of the solutions.

Once this is settled, our main goal is to investigate the relationship between the validity of Sobolev inequalities on $M$ and $\mathrm{L}^{p}-\mathrm{L}^{q}$ regularizing properties of the evolution considered. The study of the regularization of solutions occupies two sections: Section 4 contains the proof of the smoothing effect a manifold in which the Sobolev inequality holds; a certain converse is proved in Section 5. In fact, we shall say that $M$ supports an Euclidean-type Sobolev inequality if the inequality

$$
\|u\|_{2 d /(d-2)} \leq C\|\nabla u\|_{2}
$$

( $\nabla$ denotes the Riemannian gradient), holds true for all $u$ in the Sobolev space $H^{1}(M)$. If such condition holds it is know that $M$ is nonparabolic, i.e. there exists a finite (minimal) positive Green function for the Laplace-Beltrami operator on $M$. We shall show that, if $u$ is a solution to the equation $\partial_{t} u=\Delta u^{m}$ and if $M$ supports the Sobolev inequality then the fundamental smoothing property

$$
\|u(t)\|_{q} \leq C t^{-\mu}\left\|u_{0}\right\|_{p}^{\gamma}
$$

holds true for some $q>p$ and appropriate $\mu(p, q), \gamma(p, q)$, hence for a whole appropriate range of such $q, p$. If $m$ is sufficiently close to one, the converse holds; in such case the Sobolev inequality and the fundamental smoothing property are equivalent. The case $q=\infty$ is included, hence the above bound gives in particular a pointwise upper bound for the solution $u$.

We recall that a Sobolev inequality on a Riemannian manifold $M$ is known to be equivalent to a number of analytic and/or geometric properties, some of which will be recalled shortly below. We just comment here that Sobolev inequalities hold on Cartan-Hadamard manifolds, the latter being defined as those Riemannian manifolds which are simply connected and of negative curvature. The basic example we have in mind is the hyperbolic space $\mathbb{H}^{n}$ and simply connected coverings of it. On $\mathbb{H}^{n}$, the curvature is indeed constant and strictly negative.

As a motivating example of the above results, we shall consider the following situation taken from the setting of linear evolution equations. Let $L$ be the generator of a (linear) Dirichlet form $\mathcal{E}$, and let $\left\{T_{t}: t \geq 0\right\}$ be the Markov semigroup associated to it. It is a familiar fact that the validity of a bound of the form

$$
\left\|T_{t} u\right\|_{\infty} \leq C t^{-\mu}\|u\|_{q} \quad \forall t>0
$$


is equivalent, at least when a suitable bound on $\mu$ holds, to the Sobolev inequality

$$
\|u\|_{r} \leq C \mathcal{E}(u)
$$

where $r$ is explicitly related to $\mu$. This is of particular importance when dealing with the semigroup associated to a second-order linear differential operator in divergence form since it allows to link a contractivity property of the associated semigroup to a Sobolev inequality only, and hence shows that regularity of coefficients is not the key issue for contractivity properties of the semigroup to hold.

Certain analogues of this kind of correspondence have been investigated in several nonlinear setting in the papers [10], [3], [4] [5]. In particular, appropriate Sobolev inequalities are shown there to imply contractivity properties for the nonlinear semigroups associated to the porous media equations, to the evolution driven by the $p$-Laplacian $(p>2)$ and to doubly nonlinear evolution equations. None of such papers deals with singular evolution equations as the present paper does. The existence questions had not been properly examined either.

It should be remarked that the fundamental smoothing property $\|u(t)\|_{\infty} \leq t^{-\mu}\|u\|_{q}^{\gamma}$ can be proved, in the Euclidean setting and for the above mentioned equations, by other and older methods not based on the Sobolev inequality. To our knowledge, however, such methods do not easily allow to discuss even the case in which, for example, the Laplacian appearing e.g. in the porous media equation is replaced by a uniformly elliptic second order differential operator in divergence form. The main interest of the above mentioned papers lies therefore in the fact that the above fundamental smoothing property is linked to a functional property of the generator of the evolution, i.e. to a suitable Sobolev inequality and hence to geometrical properties of the manifold, a path followed in the central part of the present paper in what concerns the singular evolution equation FDE.

A new issue is addressed in Section 6, where we investigate the validity and consequences of the Poincaré inequality

$$
\|u\|_{2} \leq C\|\nabla u\|_{2},
$$

a property which may or may not hold on a manifold $M$ as before. A sufficient condition for it to hold is that the sectional curvature is smaller than $k<0$, and thus it holds e.g. in the hyperbolic space. Such condition has a spectral nature, since it amounts to the fact that the $\mathrm{L}^{2}$ spectrum of $\Delta$ is bounded away from zero, necessarily implies that the volume of intrinsic balls grows faster then polynomially (cd. [11], Chapter 5), and makes some qualitative properties of the evolution drastically different from the ones holding in the Euclidean case. In fact, we shall show that it implies that solutions corresponding to $\mathrm{L}^{q}$ data, for a suitable range of $q$ depending on $m$ vanish in finite time for any $m<1$, and give estimates both from above and from below on the extinction time. The properties of extinction in finite time are well-known both on the Euclidean space when $m$ is sufficiently small, $m<(d-2) / d$, and for any $m<1$, when the problem is posed on a bounded subset of $\mathbb{R}^{d}$ with zero boundary conditions, see e.g. [17] and [18]. We draw the attention of the reader to the fact that finite time extinction is a property that contradicts in the strongest way the property of conservation of the total mass, $\int_{M} u(t, x) \mathrm{d} x=c$ for all $t \geq 0$, that is basic property of the heat equation flow in $\mathbb{R}^{d}$ and is also satisfied by porous medium flows.

Finally, Section 7 contains a number of results on flow on Cartan-Hadamard manifolds, in particular a weak principle of local conservation of mass, which uses properties of certain cut-off functions proved by studying the Laplacian of the Riemannian distance, and its application to lower bounds on the extinction time in term of local $\mathrm{L}^{1}$ norms of the initial datum. The cases of polynomial and, respectively, exponential volume growth are discussed. Let us remark that the triviality of the topology of Cartan-Hadamard manifolds and the fact that "radial coordinates" can be defined globally, make 
such manifolds the ideal test-bed to extend analytic arguments holding in the Euclidean case, but the presence of a non trivial curvature makes this extension interesting enough. One may have a flavour of this procedure in the linear situation by noticing the deep differences between the heat kernel on the hyperbolic space and the Euclidean one.

The paper starts with a preliminary section that contains a summary of notations and of the terminology used hereafter, and recalls some facts about Sobolev inequalities on manifolds. A subsection deals with needed material on the relations between the assumed Sobolev inequalities and certain logarithmic Sobolev inequalities.

\section{Notations and preliminaries}

Throughout the paper, $M$ denotes a smooth Riemannian manifold endowed with a metric $g$. The dimension of $M$ is denoted by $d$ and assumed to be not smaller than 3 . The Riemannian gradient is indicated by $\nabla$, the Laplace-Beltrami operator by $\Delta=\Delta_{g}$, and the Riemannian measure simply by $\mathrm{d} x$. The Riemannian volume of $M$ will be always assumed to be infinite.

We refer to Hebey's book [14] for an excellent discussion of the validity of Sobolev inequalities on a manifold, and we shall extract from there (see in particular its Section 8) some results relevant for what follows. We shall in particular be concerned with the Euclidean-type Sobolev inequality

$$
\|u\|_{2 d / d-2} \leq C\|\nabla u\|_{2} .
$$

A known fact, due to Carron, is that the above Sobolev inequality is equivalent to the Faber-Krahn inequality, i.e., to the fact that for any bounded and regular open set $\Omega \subset M$, and denoting by $\lambda_{1}^{D}(\Omega)$ the bottom of the spectrum of $-\Delta$ with Dirichlet boundary conditions on the boundary of $M$, one has

$$
\lambda_{1}^{D}(\Omega) \geq C \operatorname{vol}(\Omega)^{-2 / d}
$$

We recall next that $M$ is said to be nonparabolic if it admits a (minimal) positive Green function $G$ for $\Delta$. In such case

$$
G(x, y)=\sup _{\Omega: x \in \Omega} G_{x}^{\Omega}(y)
$$

where $G_{x}^{\Omega}(y)$ is the Green function of the Dirichlet Laplacian on the regular, bounded domain $\Omega \subset M$. It can be shown that the latter supremum is either everywhere finite or everywhere infinite, and in the latter case the manifold is said to be parabolic. Conditions for parabolicity and nonparabolicity can be found in [14], pg. 230, and references quoted. The key Theorem we shall need here is the following, again due to Carron [9] (see [14], pg. 230).

Theorem 2.1 Let $M$ be a smooth Riemannian manifold of dimension $d \geq 3$ and of infinite volume. Then the validity of (2.1) is equivalent to the following facts: $M$ is nonparabolic and

$$
\operatorname{vol}(y: G(x, y)>t) \leq C t^{-d /(d-2))} .
$$

An important implication contained in the above Theorem is that the validity of (2.1) implies that $M$ is nonparabolic. This will be what we shall need to prove existence for the equations we shall be concerned with.

An important explicit class of manifolds in which (2.1) holds is given in the following Theorem: 
Theorem 2.2 ([14], pg. 232) The Sobolev inequality (2.1) is valid on any smooth, complete, simply connected manifold of nonpositive sectional curvature.

In particular, all such manifolds are nonparabolic. In fact, Theorem 8.3 of [14] states that, under the conditions of the above Theorem, an Euclidean type Sobolev inequality in $W^{1,1}$ holds. This is well known to imply the validity of all Euclidean-type Sobolev inequalities in $W^{1, q}, q>1$.

\subsection{Logarithmic Sobolev Inequalities}

It is well known that the validity of a Sobolev Inequality (SI) on a manifold is equivalent to the validity of a whole family of Gagliardo-Nirenberg Inequalities (GNI) and Logarithmic Sobolev Inequalities (LSI) as well. In the sequel, we shall use the notation

$$
J(q, u):=\int_{M} \frac{|u|^{q}}{\|u\|_{q}^{q}} \log \left(\frac{|u|}{\|u\|_{q}}\right) \mathrm{d} x .
$$

The functional $J$ is often called an entropy, or a Young functional.

It is known that the Sobolev inequality we are starting from is equivalent to a GNI and LSI, as stated in Prop. 10.3 of [1]. We recall hereafter a reduced version of that general proposition, in the form in which we shall use below.

Proposition 2.3 (Logarithmic Sobolev Inequalities) For any $0<r<2 d /(d-2)$ and $f \in W_{0}^{1,2}(M)$, the following LSI

$$
r J(r, f) \leq C_{r, d} \log \left(\mathcal{L}_{2} \frac{\|\nabla f\|_{2}^{2}}{\|f\|_{r}^{2}}\right),
$$

where $C_{r, d}=r d /(2 d-r(d-2))>0$, and $\mathcal{L}_{2}=\mathcal{L}_{2}(d, M)>0$ holds true if and only if $M$ is a Riemannian manifold on which the following Sobolev Inequality holds true:

$$
\|f\|_{2 d /(d-2)} \leq S_{2}\|\nabla f\|_{2} .
$$

Proposition 2.4 ( $\varepsilon$-Family of Logarithmic Sobolev Inequality)

Let $M$ be a Riemannian Manifold of dimension $d \geq 3$ such that the SI (2.6) holds. Then the following Family of LSI holds true for any $\varepsilon>0$ and any $f \in W_{0}^{1,2}(M)$ :

$$
\|\nabla f\|_{2}^{2} \geq \frac{\|f\|_{r}^{2}}{C_{r, d} \mathcal{L}_{2} \varepsilon}\left[r J(r, f)+C_{r, d} \log \varepsilon\right] .
$$

Proof. Just use the LSI (2.5) together with the numerical inequality $\log x \leq-\log \varepsilon+\varepsilon x$.

\section{$3 \quad$ Existence of Solutions}

In this section we discuss existence results for the Cauchy problem for the FDE in a slightly more general form. We consider the problem

$$
\begin{cases}u_{t}=\Delta(\varphi(u)) & \text { in }(0,+\infty) \times M \\ u(0, x)=u_{0}(x) & \text { in } M\end{cases}
$$


where $\varphi: \mathbb{R} \rightarrow \mathbb{R}$ is a continuous, smooth and strictly increasing function. We assume moreover that $\varphi^{\prime}>0, \varphi( \pm \infty)= \pm \infty$ and $\varphi(0)=0$. The equation is posed on a nonparabolic Riemannian Manifold $(M, g)$ and $\Lambda=-\Delta_{g}$ is the related Laplace-Beltrami operator. As remarked in the previous section, the nonparabolicity of $M$ ensures the existence of a nontrivial Green operator $G=\Lambda^{-1}$, which gives the canonical isomorphism from $H^{1}(M)$ to $H^{-1}(M)$. We take $\mathcal{H}=H^{-1}(M)$, with the usual inner product

$$
\langle f, g\rangle_{H^{-1}}=\left\langle\Lambda^{-1} f, g\right\rangle_{H^{1} \times H^{-1}}
$$

In order to construct solutions of Problem (3.1) we are going to use the theory of semigroups in Hilbert spaces generated by the subdifferentials of convex functions, as used by Brezis [8] in the Euclidean case and adapted in [17] for compact manifolds. Here is how it works: let $j$ be a lower semi-continuous function $j: \mathbb{R} \rightarrow \mathbb{R} \cup\{+\infty\}$, with $j$ not identically infinity, and let $\varphi=\partial j$ be the subdifferential of $j$. Assume also that $R(\varphi)=\mathbb{R}$. For $u \in H^{-1}(M)$ we define

$$
\Phi(u)=\int_{M} j(u) \mathrm{d} x
$$

whenever $u \in \mathrm{L}^{1}(M)$ and $j(u) \in \mathrm{L}^{1}(M)$, and define $\Phi(u)=+\infty$ otherwise. Then

Proposition 3.1 The function $\Phi$ is convex and lower semi-continuous in $\mathcal{H}=H^{-1}(M)$ so that its subdifferential is a maximal monotone operator in $\mathcal{H}$. This subdifferential $\partial \Phi$ is characterized as follows $: f \in \partial \Phi(u)$ iff

$$
G f(x) \in \varphi(u(x)) \quad \text { a.e. in } M \text {. }
$$

Recall that $G=\Lambda^{-1}$. This result has been proved by Brezis in [8], Theorem 17, and the proof found there also holds in our nonparabolic setup. The result becomes clearer when $\varphi$ is single valued so that we can write $G f(x)=\varphi(u(x))$ in the form

$$
f(x)=-\Delta \varphi(u(x)) \quad \text { a.e. in } M,
$$

which is the differential operator associated to the generalized FDE. Moreover, also Corollary 31 of [8] holds and can be reformulated as follows.

Theorem 3.2 For every $u_{0} \in H^{-1}(M)$ there exists a unique strong solution $u \in C\left([0, T]: H^{-1}(M)\right)$ of Problem 3.1 for every $T>0$. We have

$$
t \varphi(u) \in \mathrm{L}^{\infty}\left(0, T: H^{-1}(M)\right), \quad t \partial_{t} u \in \mathrm{L}^{\infty}\left(0, T: H^{-1}(M)\right) .
$$

We also have $u \varphi(u) \in L^{1}\left(Q_{T}\right)$. The solution maps $S_{t}: u_{0} \mapsto u(t)$ define a semigroup of (non-strict) contractions in $\mathcal{H}$, i. e.,

$$
\|u(t)-v(t)\|_{\mathcal{H}} \leq\|u(0)-v(0)\|_{\mathcal{H}},
$$

which turns out to be also compact in $\mathcal{H}$. There are some useful estimates: thus, when the initial datum $u_{0}$ is in $\mathrm{L}^{p}, p \geq 1$, then for any $t>0$

$$
\|u(t)\|_{p} \leq\left\|u_{0}\right\|_{p}
$$

Moreover, for $p=1$ we have $L^{1}$-contraction: for any two solutions $u, v$ we have the contraction property

$$
\|u(t)-v(t)\|_{1} \leq\|u(0)-v(0)\|_{1},
$$


Notice that $T$ is arbitrary, i.e., the solution is global in time, defined in $Q=M \times(0, \infty)$. Here are some interesting estimates: we have

$$
\frac{d}{d t}\|u\|_{\mathcal{H}}^{2}=-2 \int_{M} u \varphi(u) \mathrm{d} x
$$

for a.e. $t$ and in distribution sense. This computation implies not only decay but also regularization since it implies that $u \varphi(u)$ is controlled in $L^{1}(Q)$ by $\left\|u_{0}\right\|_{H^{-1}}$. It can be improved into a computation for the difference of two solutions $u_{1}, u_{2}$ with data $\left(u_{01}, f_{1}\right)$ and $\left(u_{02}, f_{2}\right)$ resp. We get

$$
\frac{1}{2} \frac{d}{d t}\left\|u_{1}-u_{2}\right\|_{\mathcal{H}}^{2}=-2 \int\left(u_{1}-u_{2}\right)\left(\varphi\left(u_{1}\right)-\varphi\left(u_{2}\right)\right) d x,
$$

which immediately implies uniqueness and the contraction estimate in $\mathcal{H}$. We refrain from further details because similar computations have been done in the case of a bounded domain in Euclidean space in Brezis' paper [8] and in Chapters 6, 10 and 11 of [17].

We want to obtain for our semigroup the usual properties that are known for the solutions of diffusion equations in the Euclidean case. It is convenient to consider a first step the more favorable case when $\varphi$ is smooth and $\varphi^{\prime}(u)>0$ for all $u$ and $u_{0} \in L^{2}(M) \cap L^{\infty}(M)$ and continuous. Then, the local regularity theory as in [16] and [12] shows that the solutions are classical and smooth and the initial data are taken up in a continuous way. Besides, the Comparison Principle holds in the sense that for two weak solutions $u, v$ with initial data $u_{0} \leq v_{0}$ a.e. in $M$, then $u \leq v$ a.e. in $Q$. In particular, $u_{0} \geq 0$ implies $u(t) \geq 0$ for all $t>0$. All of this can be proved just as in the Euclidean case treated in Chapter 3 of [17].

\subsection{The problem in domain with boundary and Dirichlet data}

The above technique can also be used to solve the Cauchy-Dirichlet problem on a compact smooth connected Riemannian manifold of dimension $d \geq 3$, with smooth boundary $\partial M$ when homogeneous Dirichlet boundary conditions $(u=0)$ at the boundary are assumed. We are thinking of a Riemannian ball $M^{\prime}$ in the original unbounded manifold $M$. Exactly the same results hold with the same proofs.

In this setting we can easily prove an energy inequality: assuming that $\psi\left(u_{0}\right) \in \mathrm{L}^{1}\left(M^{\prime}\right)$ for all $T>0$ we have

$$
\int_{0}^{T} \int_{M^{\prime}}|\nabla \varphi(u(t, x))|^{2} \mathrm{~d} x \mathrm{~d} t+\int_{M^{\prime}} \Psi(u(T, x)) \mathrm{d} x \leq \int_{M^{\prime}} \Psi\left(u_{0}(x)\right) \mathrm{d} x
$$

where $\Psi$ is the primitive of $\varphi$, defined as:

$$
\Psi(s)=\int_{0}^{s} \varphi(s) d s .
$$

Note that $0 \leq|\Psi(s)| \leq s \varphi(s)$. The formula is first proved as an equality for the case of good $\varphi$. Indeed, we multiply the equation by $\varphi(u)$ and integrate by parts. This can be justified exactly as it has been done in the Euclidean case in Chapter 3 of [17].

For the solutions with general $\varphi$ we have to approximate with smoother $\varphi$ and pass to the limit using the uniform boundedness given by energy estimates. In this case there is no real difference with the Euclidean case treated in Chapter 5 of [17]. Since this is not our main goal, we leave the details of the convergence of the semigroups to the interested reader. 


\subsection{Weak solutions}

In the case where $\varphi$ is a more general monotone function, we would like to recover a solution in some more standard weak sense and keep most of the extra properties we have just stated, both in the case of an unbounded manifold and the case of a compact manifold with boundary. To be specific, let us consider the case of the infinite manifold. It seems that having a strong solution should be more than just having a weak solution in the standard sense that we state below, but the context is deceiving: strong means here $H^{-1}$-strong, i.e., that $u_{t} \in H^{-1}(M)$ for a.e. $t>0$, and this is what weak solutions of the FDE are, because of the equation $u_{t}=\nabla F$, with $F=\nabla \varphi(u) \in \mathrm{L}^{2}(M)$. A convenient concept of weak solution is as follows:

Definition 3.3 (Weak Solution) A weak solution of Problem $(3.1)$ in $Q_{T}=(0, T) \times M$ is a locally integrable function $u \in C\left([0, T): L^{1}(M)\right)$, such that

(i) $\varphi(u) \in \mathrm{L}_{\text {loc }}^{1}\left(Q_{T}\right), \nabla \varphi(u) \in \mathrm{L}_{\text {loc }}^{2}\left(0, T: L^{2}(M)\right)$

(ii) The identity

$$
\int_{0}^{T} \int_{M}\left[\nabla \varphi(u) \cdot \nabla \eta+u \eta_{t}\right] \mathrm{d} x \mathrm{~d} t=0
$$

holds for any test function $\eta \in C_{c}^{1}\left(Q_{T}\right)$.

(iii) $u(0)=u_{0}$.

We can then repeat the approximation process of Chapter 5 of [17] to construct a weak solution for more general $\varphi$ and $u_{0}$. This is the result we get

Theorem 3.4 Let us consider the Cauchy Problem 3.1 with initial data $u_{0} \in \mathrm{L}^{1}(M) \cap H^{-1}(M)$. The solution constructed in Theorem 3.2 satisfies:

(i) $u$ is a global weak solution defined in $Q$. We have $\Psi(u) \in \mathrm{L}^{\infty}\left(0, \infty: \mathrm{L}^{1}(M)\right)$ and $\nabla \varphi(u) \in \mathrm{L}^{2}(Q)$.

(ii) $u$ belongs to the space $C\left([0, \infty): \mathrm{L}^{1}(M)\right)$ and takes the initial data $u_{0}$.

(iii) For any two solutions $u, v$ we have the $L^{1}$-contraction property (3.7).

(iv) The energy inequality (3.9) holds.

(v) If $u_{0} \geq 0$ then $u(t) \geq 0$ for all $t>0$.

(vi) Comparison Principle. If $u, v$ are two weak solutions as constructed in the theorem by approximation, and they have initial data $u_{0} \leq v_{0}$ a.e. in $M$, then $u \leq v$ a.e. in $Q$.

(vii) If moreover the initial datum $u_{0}$ is in $\mathrm{L}^{p}(M)$ with $p \geq 1$, then for any $t>0$

$$
\|u(t)\|_{p} \leq\left\|u_{0}\right\|_{p}
$$

In particular, essentially bounded initial data produce essentially bounded solutions.

Details about to the proofs can be obtained from the Euclidean case in a bounded domain treated in Chapters 5, 6 of [17] and the case of the whole space $\mathbb{R}^{d}$ treated in Chapter 9. It is just a question of deriving the results for the case of $\operatorname{good} \varphi$ and a Riemannian ball and passing to the limit using the uniform estimates to recover the solution of Theorem 3.2.

In the case of nonnegative solutions with data in some $\mathrm{L}^{p}$ space, we can pass to the limit in the problems in bounded domains to find the solution on the whole manifold in a simpler way. If $u_{n}$ is the 
solution on $M_{n}$ defined above, after extending to zero outside $M_{n}$, we can check that the $u_{n}$ converge in a monotonically nondecreasing way to $u$, the solution of the Cauchy problem, and thus for any $p \geq 1$, we get

$$
u_{n}^{p}(t, x) \nearrow u^{p}(t, x)
$$

for almost any $t, x$. The Monotone Convergence Theorem implies that

$$
\int_{M} u_{n}^{p}(t, x) \mathrm{d} x \rightarrow \int_{M} u^{p}(t, x) \mathrm{d} x
$$

for a.e. $t$. These ideas are convenient when we want to reduce estimates in the unbounded manifold to estimates on balls.

Remarks. (1) We can even dispense with the requirement that $u_{0} \in H^{-1}(M)$ in the assumptions of Theorem 3.4 and still get a solution for every $u_{0} \in L^{1}(M)$ by using the $L^{1}$-contractivity property, (3.7). This solution is in principle only a limit solution in the sense of [17], Section 6.1, or a mild solution in the sense of [2], but the smoothing effect that we shall prove, Theorem 4.1, makes it a weak solution since for any $t>0$ we have $u(t) \in L^{1} \cap L^{\infty} \subset L^{2}$. Same for $L^{p}$ data.

(2) The technique can be extended to solve the non-homogeneous problem

$$
u_{t}-\Delta \varphi(u)=f
$$

provided for instance that the term $f$ is absolutely continuous from $(0, T)$ to $H^{-1}(M)$.

(3) We are not addressing here the issue of strict positivity of solutions with nonnegative data, a topic strictly related to the validity of the Harnack inequality for the evolution considered.

\section{Smoothing effects}

We concentrate from now on the Cauchy Problem for the Fast Diffusion Equation:

$$
\begin{cases}u_{t}=\Delta\left(u^{m}\right) & \text { in }(0,+\infty) \times M \\ u(0, x)=u_{0}(x) & \text { in } M\end{cases}
$$

where $0<m<1$. We comment here that exactly the same results hold with the same proof for the Cauchy-Dirichlet problem on an incomplete, smooth manifold, with homogeneous Dirichlet boundary conditions on $\partial M$. For signed solutions $u^{m}$ means $|u|^{m-1}$.

Our first result states that when the Sobolev inequality holds, the fundamental smoothing effect holds as well.

Theorem 4.1 (Smoothing Effect) Let $M$ be a Riemannian manifold of dimension $d \geq 3$ and infinite volume, and assume that the Sobolev inequality (2.1) holds. Let $u(t)$ be a weak solution to Problem (4.1). Then, for any $q>p_{c}, q \geq 1, p_{c}=d(1-m) / 2$ we have:

$$
\|u(t)\|_{\infty} \leq C \frac{\left\|u_{0}\right\|_{q}^{\gamma}}{t^{\alpha}}
$$

where

$$
\alpha=\frac{d}{2 q-d(1-m)}, \quad \gamma=\frac{2 q}{2 q-d(1-m)} .
$$


Remark 4.2 The estimates (4.2) are identical to those which are well-known to hold in the Euclidean setting, both as regards their form and as regards the bound on q, cf. [18].

Proof of Theorem 4.1. By the Maximum Principle, we only need to prove the estimates for nonnegative solutions. First we recall, for the reader's convenience, some standard properties of the time derivative $\mathrm{L}^{p}$ norm of the solution to the problem (4.1).

Lemma 4.3 Let $u$ be a nonnegative weak solution to the problem (4.1) corresponding to an initial datum $u_{0} \in \mathrm{L}^{1}(M) \cap \mathrm{L}^{\infty}(M)$, and let $m>0, p>p_{c}, p \geq 1, p_{c}=d(1-m) / 2, t \geq 0$. Then for all $p \geq p_{0}$ and a.e. $t>0$ we have

$$
-\frac{d}{d s} \int_{M} u(s, x)^{p} \mathrm{~d} x \geq \frac{4 p(p-1) m}{\theta^{2}}\left\|\nabla\left(u(s)^{\theta / 2}\right)\right\|_{2}^{2}
$$

where $\theta=p+m-1$. Moreover, letting $p:[0, t) \rightarrow[q,+\infty]$, q as stated, be a $C^{1}$ nondecreasing function such that $p(0)=q$ and letting $\theta(s)=p(s)+m-1$ then gives

$$
\frac{\mathrm{d}}{\mathrm{d} s} \log \|u(s)\|_{p(s)}=\frac{\dot{p}(s)}{p(s)} J(p(s), u(s))-\frac{4 m(p(s)-1)}{\theta(s)^{2}} \frac{\left\|\nabla\left(u^{\theta(s) / 2}\right)\right\|_{2}^{2}}{\|u(s)\|_{p(s)}^{p(s)}} .
$$

The result shows how the Young functional (2.4) enters naturally in our setting.

The first estimate is proved in a formal way by multiplying the equation by $u^{p-1}$, integrating in space and then integrating by parts. In order to justify the calculation we use the approximation process outlined in the previous section, first by a problem posed in a ball with zero Dirichlet conditions, and then by approximating the nonlinearity of the data. In this, since $u \geq 0$ we only need to approximate the data by adding $\epsilon=1 / k>0$ both at $t=0$ and on the boundary. See similar calculation in Section 5.3 of [17].

The second part of the proof proceeds exactly as in the proof of Lemmas 3.1, 3.2 and 3.3 of [4] where the porous medium equation has been considered; this is formally the same equation that we consider here, but with $m>1$ and the proof presented there holds for any $m>0$.

In the next lemma we are going to use the $\varepsilon$-family of LSI (2.7) in order to estimate the energy in terms of the entropy, so that we finally get a closed differential inequality for the logarithm of the $\mathrm{L}^{p}$ norm.

Lemma 4.4 Let $u$ be a weak solution to Problem (4.1) corresponding to an initial datum $u_{0} \in \mathrm{L}^{1}(M) \cap$ $\mathrm{L}^{\infty}(M)$. Let $p:[0, t) \rightarrow[q,+\infty]$, be a $C^{1}$ non-decreasing function such that $p(0)=q>p_{c}, q \geq 1$, $p_{c}=d(1-m) / 2$, and let $\theta(s)=p(s)+m-1$. Then the following closed differential inequality holds true:

$$
\begin{aligned}
\frac{\mathrm{d}}{\mathrm{d} s} \log \|u(s)\|_{p(s)} & \leq \frac{\dot{p}(s)}{p^{2}(s)}(1-m) C_{p, d} \log \|u(s)\|_{p(s)} \\
& -\frac{\dot{p}(s)}{p^{2}(s)} C_{p, d} \log \left[\frac{4 m p(s)^{2}(p(s)-1)}{C_{p, d} \mathcal{L}_{2} \theta(s)^{2} \dot{p}(s)}\right]
\end{aligned}
$$

where $C_{p, d}=d p(s) /(2 p(s)-d(1-m))>0$, and $\mathcal{L}_{2}>0$ is the constant in the LSI (2.5). 
Proof. First we use the $\varepsilon$-LSI (2.7) to estimate the last term of (4.5), taking advantage of the freedom of choice of the parameter $r$, which will be chosen later:

$$
\begin{aligned}
\frac{\mathrm{d}}{\mathrm{d} s} \log \|u(s)\|_{p(s)} & =\frac{\dot{p}(s)}{p(s)} J(p(s), u(s))-\frac{4 m(p(s)-1)}{\theta(s)^{2}} \frac{\left\|\nabla\left(|u|^{\theta(s) / 2}\right)\right\|_{2}^{2}}{\|u(s)\|_{p(s)}^{p(s)}} \\
& \leq \frac{\dot{p}(s)}{p^{2}(s)} J\left(1,|u(s)|^{p(s)}\right)-\frac{4 m(p(s)-1)}{C_{r, d} \mathcal{L}_{2} \varepsilon \theta(s)^{2}} \frac{\left\|\left.u\right|^{\theta(s) / 2}\right\|_{r}^{2}}{\|u(s)\|_{p(s)}^{p(s)}} \\
& \times\left[r J\left(r,|u(s)|^{\theta(s) / 2}\right)+C_{r, d} \log \varepsilon\right] \\
& =\frac{\dot{p}(s)}{p^{2}(s)} J\left(1,|u(s)|^{p(s)}\right)-\frac{4 m(p(s)-1)}{C_{r, d} \mathcal{L}_{2} \varepsilon \theta(s)^{2}} \frac{\|u(s)\|_{\theta(s) r / 2}^{\theta(s)}}{\|u(s)\|_{p(s)}^{p(s)}} \\
& \times\left[J\left(1,|u(s)|^{\theta(s) r / 2}\right)+C_{r, d} \log \varepsilon\right]
\end{aligned}
$$

where we used the obvious identity facts that $p J(p, u)=J\left(1,|u|^{p}\right)$ and the fact that $\left\||u|^{\theta(s) / 2}\right\|_{r}^{2}$ $=\|u(s)\|_{\theta(s) r / 2}^{\theta(s)}$. Now we choose

$$
\varepsilon=\frac{4 m p(s)^{2}(p(s)-1)}{C_{r, d} \mathcal{L}_{2} \theta(s)^{2} \dot{p}(s)} \frac{\|u(s)\|_{\theta(s) r / 2}^{\theta(s)}}{\|u(s)\|_{p(s)}^{p(s)}}=\varepsilon_{1} \frac{\|u(s)\|_{\theta(s) r / 2}^{\theta(s)}}{\|u(s)\|_{p(s)}^{p(s)}}
$$

and we get that

$$
\begin{aligned}
\frac{\mathrm{d}}{\mathrm{d} s} \log \|u(s)\|_{p(s)} & \leq \frac{\dot{p}(s)}{p^{2}(s)}\left[J\left(1,|u(s)|^{p(s)}\right)-J\left(1,|u(s)|^{\theta(s) r / 2}\right)\right] \\
& -\frac{\dot{p}(s)}{p^{2}(s)} C_{r, d} \log \frac{\|u(s)\|_{\theta(s) r / 2}^{\theta(s)}}{\|u(s)\|_{p(s)}^{p(s)}}-\frac{\dot{p}(s)}{p^{2}(s)} C_{r, d} \log \varepsilon_{1}
\end{aligned}
$$

It is at this point that we choose the parameter $r$ explicitly. In fact, set $r=2 p(s) / \theta(s)$ and notice that $2 \leq r<2 d /(d-2)$, since we assumed $m \in(0,1)$ and $p(s)>p_{c}=d(1-m) / 2$. With this choice of $r$, inequality (4.8) becomes:

$$
\begin{aligned}
\frac{\mathrm{d}}{\mathrm{d} s} \log \|u(s)\|_{p(s)} & \leq \frac{\dot{p}(s)}{p^{2}(s)}\left[J\left(1,|u(s)|^{p(s)}\right)-J\left(1,|u(s)|^{p(s)}\right)\right] \\
& -\frac{\dot{p}(s)}{p^{2}(s)} C_{r, d} \log \frac{\|u(s)\|_{p(s)}^{\theta(s)}}{\|u(s)\|_{p(s)}^{p(s)}}-\frac{\dot{p}(s)}{p^{2}(s)} C_{r, d} \log \varepsilon_{1} \\
& =-\frac{\dot{p}(s)}{p^{2}(s)} C_{r, d} \log \|u(s)\|_{p(s)}^{m-1}-\frac{\dot{p}(s)}{p^{2}(s)} C_{r, d} \log \varepsilon_{1}
\end{aligned}
$$

and

$$
\begin{aligned}
C_{r, d}=\frac{d r}{2 d-r(d-2)} & =\frac{1}{2}\left(\frac{1}{r}-\frac{d-2}{2 d}\right)^{-1}=\frac{1}{2}\left(\frac{p(s)+m-1}{2 p(s)}-\frac{d-2}{2 d}\right)^{-1} \\
& =\frac{d p(s)}{2 p(s)-d(1-m)}>0
\end{aligned}
$$


which is strictly positive by the assumption $p(s)>p_{c}=d(1-m) / 2$. The proof is thus complete.

The previous Lemma gives a closed differential inequality for $\log \|u(s)\|_{p(s)}$ which we rephrase here.

Proposition 4.5 Let $u$ be a weak solution to (4.1) corresponding to an initial datum $u_{0} \in L^{\infty}(M)$. Let $p:[0, t) \rightarrow[q,+\infty]$, be a $C^{1}$ nondecreasing function such that $p(0)=q>p_{c}, q \geq 1, p_{c}=$ $d(1-m) / 2$, and $p(s) \rightarrow p \in(q, \infty]$ as $s \uparrow t$. Let $\theta(s)=p(s)+m-1$. If we let

$$
\begin{aligned}
& y(s)=\log \|u(s)\|_{p(s)} \\
& a(s)=-\frac{\dot{p}(s)}{p(s)} \frac{d(1-m)}{2 p(s)-d(1-m)} \\
& b(s)=\frac{\dot{p}(s)}{p(s)} \frac{d}{2 p(s)-d(1-m)}\left[\log \left(\frac{(p(s)-1)(2 p(s)-d(1-m))}{p(s) \theta(s)^{2}}\right)+\log \left(\frac{4 m p^{2}(s)}{\mathcal{L}_{2} d \dot{p}(s)}\right)\right]
\end{aligned}
$$

Then the following differential inequality holds true $\forall s \geq 0$ :

$$
\frac{\mathrm{d} y(s)}{\mathrm{d} s}+a(s) y(s)+b(s) \leq 0
$$

so that $y(s) \leq y_{L}(s)$, provided $y(0) \leq y_{L}(0)$, where

$$
y_{L}(s)=\exp \left(-\int_{0}^{s} a(\lambda) d \lambda\right)\left[y_{L}(0)-\int_{0}^{s} b(\lambda) \exp \left(\int_{0}^{\lambda} a(\eta) d \eta\right) d \lambda\right]
$$

is a solution of the ordinary differential equation

$$
\frac{\mathrm{d} y(s)}{\mathrm{d} s}+a(s) y(s)+b(s)=0
$$

and takes the form:

$$
y_{L}(t)=\frac{2 q}{2 q+d(m-1)} y_{L}(0)-\frac{d}{2 q-d(1-m)} \log (t)+C_{2}
$$

where $C_{2}$ depends on $m, d, q, p$ and on the Sobolev constant.

Proof. First we compute:

$$
\begin{aligned}
& A(s)=\int_{0}^{s} a(s) \mathrm{d} s=-\int_{0}^{s} \frac{d(1-m)}{p(\lambda)[2 p(\lambda)-d(1-m)]} \dot{p}(\lambda) \mathrm{d} \lambda=-\log \frac{p(0)[2 p(\lambda)-d(1-m)]}{p(\lambda)[2 p(0)-d(1-m)]}, \\
& \mathrm{e}^{-A(t)}=\lim _{s \uparrow t} \mathrm{e}^{-A(s)}=\frac{2 p(0)}{2 p(0)-d(1-m)}=\frac{2 q}{2 q-d(1-m)}
\end{aligned}
$$

we remark that this integral is independent of the particular choice of $p$, with the running assumption on $p$. Now we calculate

$$
\begin{aligned}
B(s) & =\int_{0}^{s} b(\lambda) \mathrm{e}^{A(\lambda)} \mathrm{d} \lambda=\frac{d[2 q-d(1-m)]}{q} \int_{0}^{s} \frac{\dot{p}(\lambda)}{[p(\lambda)-d(1-m)]^{2}}\left[\log \left(\frac{4 m p^{2}(\lambda)}{\mathcal{L}_{2} d \dot{p}(\lambda)}\right)\right. \\
& \left.+\log \left(\frac{(p(\lambda)-1)(2 p(\lambda)-d(1-m))}{p(\lambda)[p(\lambda)+m-1]^{2}}\right)\right] \mathrm{d} \lambda=\frac{d[2 q-d(1-m)]}{2 q}\left[B_{1}(s)+B_{2}(s)\right]
\end{aligned}
$$


Let now $p(s)=q t /(t-s)$. A tedious but straightforward calculation, which uses the fact that $p^{\prime} / p^{2}=q t$, shows that

$$
B_{1}(s)=\log \left(\frac{4 m q t}{\mathcal{L}_{2} d}\right)\left[\frac{1}{2 q-d(1-m)}-\frac{1}{2 p(s)-d(1-m)}\right] .
$$

Analogous calculations lead to

$$
B_{2}(s)=\int_{q}^{p(s)} \frac{1}{[2 \eta-d(1-m)]^{2}} \log \left(\frac{(\eta-1)(2 \eta-d(1-m))}{\eta[\eta+m-1]^{2}}\right) \mathrm{d} \eta
$$

we just remark that the value of $B_{2}$ depends only on $m, d, q$ and $p(s)$, moreover $B_{2}$ remains bounded also when $p(s) \rightarrow \infty$. We can thus conclude that

$$
y_{L}(s)=\frac{q[2 p(s)-d(1-m)]}{p(s)[2 q-d(1-m)]} y_{L}(0)-\frac{d[p(s)-q]}{p(s)[2 q-d(1-m)]} \log (t)+C_{1}
$$

where $C_{1}$ depends on $m, d, q, p(s)$ and $\mathcal{L}_{2}$, the constant in LSI $(2.5)$; moreover $C_{1}$ remains bounded when $p(s) \rightarrow \infty$.

Again we note that these integrals are independent of the explicit choice of $p(s)$

Now we let $s \rightarrow t^{-}$, so that $p(s) \rightarrow+\infty$ and we get

$$
y_{L}(t)=\frac{2 q}{2 q-d(m-1)} y_{L}(0)-\frac{d}{2 q-d(1-m)} \log (t)+C_{2}
$$

where $C_{2}$ depends on $m, d, q, p$ and on the Sobolev constant.

To complete the proof of Theorem 4.1 the procedure is standard. Write

$$
\begin{aligned}
\log \|u(t)\|_{\infty} & =\lim _{s \uparrow t} \log \|u(t)\|_{r(s)} \leq \lim _{s \uparrow t} \log \|u(s)\|_{r(s)}= \\
& =\lim _{s \uparrow t} y(s) \leq \lim _{s \uparrow t} y_{L}(s)=y_{L}(t)
\end{aligned}
$$

so that letting $y_{L}(0)=\log \|u(0)\|_{q}=y(0)$ one obtains:

$$
\|u(t)\|_{\infty} \leq \mathrm{e}^{y_{L}(t)}=\frac{\mathrm{e}^{C_{2}}}{t^{\alpha}}\|u(0)\|_{q}^{\gamma}
$$

Provided $\alpha$ and $\gamma$ are as in the statement. Removing the assumption $u_{0} \in \mathrm{L}^{\infty}$ is a standard approximation argument.

Remark 4.6 1) Interpolating between the previous bound and the contraction property $\|u(t)\|_{q} \leq$ $\left\|u_{0}\right\|_{q}$, valid for all $t>0$, yields the bound

$$
\|u(t)\|_{p} \leq C \frac{\left\|u_{0}\right\|_{q}^{\gamma}}{t^{\alpha}}
$$

where

$$
\begin{aligned}
& p \geq q, \quad q>p_{c}, \quad q \geq 1, \quad p_{c}=d(1-m) / 2 \\
& \alpha=\frac{d[p-q]}{p[2 q-d(1-m)]}, \quad \gamma=\frac{q[2 p-d(1-m)]}{p[2 q-d(1-m)]}
\end{aligned}
$$


which could also be proved along the same lines. Here $C$ depends on $m, d, q, p$ and on the constant appearing in (2.1); moreover one can show that $C$ remains bounded when $p \rightarrow \infty$ and one may recover the bound (4.2) in this way as well.

3) The same results hold for solutions to the equation $\partial_{t} u=\Delta \varphi(u)$, with an almost identical proof, provided $\varphi^{\prime}(u) \geq c u^{m-1}$ with $m$ as above.

\section{From the smoothing property to Sobolev inequalities}

The next result is a converse of the previous one.

Theorem 5.1 Let $M$ be a nonparabolic Riemannian manifold of dimension not smaller than 3 and of infinite volume, assume that $m \in\left(m_{s}, 1\right)$ where $m_{s}=(d-2) /(d+2)$. Assume moreover that the bound (4.10) holds for $p=1+m>q$ with $q>p_{c}, q \geq 1$ and for any weak solution to the equation at hand. Then the Sobolev inequality (2.1) holds.

Open problem. Does the above result hold also for $m \in\left(0, m_{s}\right]$ or there exists a manifold in which (4.10) holds for some $m$ in that range, but the Sobolev inequality does not hold?

Proof of Theorem 5.1. It is sufficient to prove that the bound

$$
\|u(t)\|_{1+m}^{1+m} \leq C \frac{\left\|u_{0}\right\|_{q}^{q+(1+m-q) \gamma}}{t^{(1+m-q) \alpha}}
$$

where $\max \left\{1, p_{c}\right\}<q<1+m$, implies a single GNI, then thanks to the results of [1] one knows that this is equivalent to the Sobolev inequality as well.

Notice that, since $m>m_{s}:=(d-2) /(d+2)$, one readily checks that $1+m>\max \left\{1, p_{c}\right\}$.

- Energy Estimates

Consider the energy identity

$$
\frac{1}{1+m} \frac{\mathrm{d}}{\mathrm{d} t}\|u(t)\|_{1+m}^{1+m}=-\left\|\nabla\left(u^{m}(t)\right)\right\|_{2}^{2}
$$

An integration over $(0, t)$ gives:

$$
\begin{aligned}
\frac{1}{1+m}\left[\|u(t)\|_{1+m}^{1+m}-\left\|u_{0}\right\|_{1+m}^{1+m}\right] & =-\int_{0}^{t}\left\|\nabla\left(u^{m}(t)\right)\right\|_{2}^{2} \mathrm{~d} t \\
& \geq-\int_{0}^{t}\left\|\nabla\left(u_{0}^{m}\right)\right\|_{2}^{2} \mathrm{~d} t=-t\left\|\nabla\left(u_{0}^{m}\right)\right\|_{2}^{2}
\end{aligned}
$$

since the $\mathrm{L}^{2}$ norm of $\nabla\left(u^{m}\right)$ is monotone in time as proved in [7]. We rewrite it in a more convenient form:

$$
\|u(t)\|_{1+m}^{1+m} \geq-t(m+1)\left\|\nabla\left(u_{0}^{m}\right)\right\|_{2}^{2}+\left\|u_{0}\right\|_{1+m}^{1+m}
$$

- Optimization

Now we are going to put together inequalities (5.3) and (5.1), to get

$$
\frac{\left\|u_{0}\right\|_{q}^{q+(1+m-q) \gamma}}{t^{(1+m-q) \alpha}} \geq\|u(t)\|_{1+m}^{1+m} \geq-t(m+1)\left\|\nabla\left(u_{0}^{m}(t)\right)\right\|_{2}^{2}+\left\|u_{0}\right\|_{1+m}^{1+m}
$$


which gives:

$$
f(t)=A t^{-\delta}+B t \geq C
$$

where

$$
A=C\left\|u_{0}\right\|_{q}^{q+(1+m-q) \gamma}, \quad B=(m+1)\left\|\nabla\left(u_{0}^{m}(t)\right)\right\|_{2}^{2}, \quad C=\left\|u_{0}\right\|_{1+m}^{1+m}
$$

The real function $f$ has a unique minimum for positive $t$, namely

$$
t=\hat{t}:=\left(\frac{\delta A}{B}\right)^{1 /(1+\delta)}
$$

and one has

$$
f(\hat{t})=K A^{1 /(1+\delta)} B^{\delta /(1+\delta)},
$$

$k$ being a positive numerical constant.

This gives, once substituting the values of $A, B$ and $C$ :

$$
\left\|u_{0}\right\|_{1+m}^{1+m} \leq K_{1}\left\|u_{0}\right\|_{q}^{\frac{q+(1+m-q) \gamma}{1+(1+m-q) \alpha}}\left\|\nabla\left(u_{0}^{m}(t)\right)\right\|_{2}^{\frac{2 \alpha(1+m-q)}{1+(1+m-q) \alpha}} .
$$

Letting $v=u_{0}^{m}$, then finally obtains the following Gagliardo-Nirenberg inequality:

$$
\|v\|_{(1+m) / m}^{(1+m) / m} \leq K_{1}\|v\|_{q / m}^{\frac{q+(1+m-q) \gamma}{m[1+(1+m-q) \alpha]}}\|\nabla v\|_{2}^{\frac{2 \alpha(1+m-q)}{1+(1+m-q) \alpha}} .
$$

As mentioned above this is well-known to be equivalent to the Euclidean-type Sobolev inequality.

\section{Poincaré inequality, lower bounds and finite extinction time}

In this section we make an additional assumption, namely, the validity of the Poincaré inequality

$$
\|u\|_{2} \leq C\|\nabla u\|_{2}, \quad \forall u \in H^{1}(M) .
$$

This inequality is equivalent to the spectral gap condition: $\inf \sigma(-\Delta) \geq 1 / C>0, \quad \sigma(\Delta)$ being the $\mathrm{L}^{2}$ spectrum of the Laplace-Beltrami operator, and thus for example it does not hold in the whole Euclidean space. It is known that (6.1) holds if $M$ is simply connected and with sectional curvature smaller than $K<0$ (see [14], Remark 8.1). Typical examples are the hyperbolic space or simply connected coverings of such space. The validity of the spectral gap necessarily implies that the volume of intrinsic balls grows faster than polynomially (cf. [11], Chapter 5).

We shall show that under these conditions a large class of solutions of the Fast Diffusion Equation vanish in finite time. We have:

Theorem 6.1 Let $M$ be a Riemannian manifold of dimension $d \geq 3$ having infinite volume, assume that the Sobolev inequality (2.1) holds and, moreover, that the gap condition (6.1) is true. Let $q>1$ be finite number, and $q \geq p_{c}$, where $p_{c}=d(1-m) / 2$. Then there exists $C>0$ depending only on $m, p$ and on the constants appearing in (6.1) and in (2.1) such that, for any weak solution $u(t)$ of Problem (4.1) with data $u_{0} \in \mathrm{L}^{q}(M)$ and any $t>s \geq 0$ we have:

$$
\|u(t)\|_{q}^{1-m} \leq\|u(s)\|_{q}^{1-m}-C(t-s) .
$$


In particular, choosing $s=0$ and letting $t$ be proportional to $\left\|u_{0}\right\|_{q}^{1-m}$ shows that $u(t)$ vanishes identically after a finite time $T\left(u_{0}\right)$, with the bound

$$
T\left(u_{0}\right) \leq \text { const. }\left\|u_{0}\right\|_{q}^{1-m} .
$$

This phenomenon happens the in Euclidean case but the difference is felt in the range of exponents where it applies. Thus, when the problem is posed in $\mathbb{R}^{d}$ no extinction takes place when $m \in\left(m_{c}, 1\right)$, $m_{c}=(d-2) / d$. In fact, our result matches exactly what is obtained for the Fast Diffusion Equation posed on a bounded domain of $\mathbb{R}^{d}$ with zero Dirichlet boundary conditions.

Proof. We shall denote by $C$ an inessential numerical constant which may change from line to line. Let $a_{q}(t):=\|u(t)\|_{q}^{q}$ for $q>1$. Then, a standard calculation allows to obtain the equality

$$
d a_{q}(t) / d t \leq-C\left\|\nabla\left(|u(t)|^{(m+q-1) / 2}\right)\right\|_{2}^{2}
$$

with equality for strong solutions. Interpolating between the Sobolev and the gap condition we have $\|f\|_{r} \leq C\|\nabla f\|_{2}$ for all $r \in[2,2 d /(d-2)]$. Therefore,

$$
d a_{q}(t) / d t \leq-C\left\||u(t)|^{(m+q-1) / 2}\right\|_{r}^{2}=-C\|u(t)\|_{(m+q-1) r / 2}^{m+q-1}
$$

Let $r=2 q /(m+q-1)$. It is immediate that $r>2$ for any choice of $q$ (since $m<1)$ and that $r \leq 2 d /(d-2)$ exactly when $q \geq p_{c}$. The above choice of $r$ is therefore allowable and it leads to the differential inequality

$$
d a_{q}(t) / d t \leq-C a_{q}(t)^{(m+q-1) / q} .
$$

Such inequality can be integrated to yield

$$
a_{q}(t)^{(1-m) / q}-a_{q}(s)^{(1-m) / q} \leq-C(t-s),
$$

or equivalently to

$$
\|u(t)\|_{q}^{1-m} \leq\|u(s)\|_{q}^{1-m}-C(t-s),
$$

which is an alternative form of our statement.

It is easy to notice, by keeping track of the constant involved, that our bound on the vanishing time diverges both when $q \rightarrow 1$ and when $q \rightarrow+\infty$. However, the above result and the $\mathrm{L}^{q}-\mathrm{L}^{\infty}$ smoothing property can be used to prove a bound in the $\mathrm{L}^{\infty}$ norm as follows:

Corollary 6.2 Suppose that the assumptions on $M$ stated in Theorem 6.1 hold, that $q>p_{c}, q \geq 1$, $p_{c}=d(1-m) / 2$, and take any data $u_{0} \in \mathrm{L}^{q}(M)$ and any $\varepsilon>0$. Then the bound

$$
\|u(t)\|_{\infty} \leq \frac{C}{\varepsilon^{\alpha}}\left[\left\|u_{0}\right\|_{p}^{1-m}-C(t-\varepsilon)\right]^{\gamma /(1-m)}
$$

holds true. In particular, $u(t)$ tends to zero in finite time in all $L^{p}$ norms, $p \in[q,+\infty]$.

Proof. Use the bound (6.2) in the time interval $[0, t-\varepsilon]$ and the bound (4.2) in the time interval $[t-\varepsilon, t]$, gluing them by means of the semigroup property.

The extinction result is not expected to be true for data in $\mathrm{L}^{q}$ spaces with $q$ smaller than $p_{c}$. The proof can be easily done in the Euclidean case by using scaling. Notice that $1 \leq q<p_{c}$ is possible only in the range $m<m_{c}=(d-2) / d$. 
Proposition 6.3 Let the problem be posed in a bounded domain $\Omega$ of $\mathbb{R}^{d}$ with zero Dirichlet conditions. If $m<m_{c}$, there exist solutions $u(t, x) \geq 0$ with data $u_{0}(x)$ in $\mathrm{L}^{q}(\Omega)$ with $1 \leq q<p_{c}$ that do not extinguish in finite time. The same happens if $m=m_{c}$ and $q=1$.

Proof. (i) CASE $m<m_{c}$. We consider the ball $B=B_{1}(0)$ and take a solution $u_{1}(t, x)$ that has bounded initial data and vanishes at a time $T>0$. Let us assume that $\int_{B} u_{1}^{q}(0, x) d x=1$. We now make a scaling transformation that preserves the equation:

$$
u_{k}(t, x)=k^{\lambda} u\left(k^{-\mu} t, k x\right), \mu=\lambda(1-m)-2 .
$$

We will choose a $\lambda>2 /(1-m)$ so that $\mu>0$. The new solution is defined on the ball $B_{k}$ of radius $R_{k}=1 / k$ and center 0 . Its initial integral is set to be

$$
\int_{B_{k}} u_{k}^{q}(0, x) d x=k^{q \lambda-d} \int_{B} u_{1}^{q}(0, x) d x=k^{q \lambda-d},
$$

We want the sum of this sequence to be finite for the choice $k=2^{n}, n=1,2, \ldots$, so that we need $\lambda<d / q$. This is possible if $d>2 q /(1-m)$, i.e., if $q<p_{c}$. Note that solution $u_{k}$ vanishes in a time $T_{k}=k^{\mu} T$ and this goes to infinity as $k \rightarrow \infty$.

Take now the solution $U$ in $B$ with initial data

$$
U^{q}(0, x)=\sum_{k=2^{n}, n=1}^{\infty} u_{k}^{q}(0, x),
$$

where the $u_{k}(0, x)$ are extended by zero outside of $B_{k}$. It is easy to see that $U(0, x)$ is an $\mathrm{L}^{q}$ integrable function. Also, a simple comparison shows that

$$
U(t, x) \geq u_{k}(t, x) \quad \forall x \in B_{k}, t>0 .
$$

It follows that $U$ does not vanish identically in finite time.

(ii) CASE $q=1, m=m_{c}$. Let us consider for $n=1,2, \ldots$ a solution $u_{n}(t, x)$ of the Cauchy problem posed on $\mathbb{R}^{d}$, for the FDE in the critical case, i.e. when $m=m_{c}$, corresponding to a positive initial datum $0<u_{0 n} \in \mathrm{L}^{1}\left(\mathbb{R}^{d}\right)$, with total mass $\int_{\mathbb{R}^{d}} u_{0 n} \mathrm{~d} x=1 / n^{2}$. It is well known that the conservation of mass holds also in the critical case, see e.g. [18]; this means in particular that these solutions do not extinguish in finite time. Now, let us fix $n$ and consider the family $u_{R}(t, x)$ of solutions of the mixed Cauchy-Dirichlet problem on the ball $B_{R}$ corresponding to the initial data $u_{0, R}=u_{0} \chi_{B_{R}}$. It is well-known that $u_{R} \rightarrow u$ as $R \rightarrow \infty$, in the topology of $C\left((0,+\infty) ; \mathrm{L}^{1}\left(\mathbb{R}^{d}\right)\right)$. The solutions of these Cauchy-Dirichlet problems may extinguish in a finite time $T\left(u_{0, R}\right)>0$. However, the limit $u_{R} \rightarrow u$ implies that for any $n \geq 1$ there exists an $R=R(n)$ such that the extinction time for $u_{R}$ is bigger than $T\left(u_{0, R}\right) \geq n$. The next step is to rescale the function $u_{R}$ with $R=R(n)$ to get:

$$
\bar{u}_{n}(t, x)=R^{n} u_{R}(t, R x)
$$

(notice that time is not involved) which is a solution of the Cauchy-Dirichlet problem on $B_{1}$, corresponding to the initial datum

$$
\bar{u}_{0 n}(x)=R^{n} u_{R}(0, R x)
$$

which has the same extinction time $T\left(\bar{u}_{0}\right)=T\left(u_{0, R}\right) \geq n$. 
Consider then the solution $U(t, x)$ of the mixed Cauchy-Dirichlet problem posed on $B_{1}$ corresponding to the initial datum

$$
U_{0}(x)=\sum_{n=1}^{\infty} \bar{u}_{0 n}
$$

where $\bar{u}_{0 n}$ as above, so that the mass of $U_{0}$

$$
0<\int_{B_{1}} U_{0}(x) \mathrm{d} x=\sum_{n=1}^{\infty} \int_{B_{1}} \bar{u}_{0, n} \mathrm{~d} x<+\infty
$$

is finite and positive. By comparison it is easy to see that

$$
U(t, x) \geq \bar{u}_{n}(t, x) \forall n,
$$

which implies that $T\left(U_{0}\right) \geq T\left(\bar{u}_{0, n}\right) \geq n$ for any $n \geq 1$. This proves that $T\left(U_{0}\right)=+\infty$ that means that $U$ does not extinguish in finite time.

The case $m_{c}<m<1$ with $q=1$ also leads to extinction. We just have to change the proof of Theorem 6.1 by considering the evolution of the integrals

$$
\widetilde{a}(t)=\int_{M} h(u(t, x)) d x
$$

where $h^{\prime}(s)=1 /\left(1+s^{2}\right)^{1 / 2+\varepsilon}, h^{\prime}(0)=0$, so that $h$ is bounded. We refrain from giving more details. The argument does not work for $m=m_{c}, q=1$.

A further Corollary of the main result of this Section involves a lower bound on $\mathrm{L}^{p}$ norms of the solution.

Corollary 6.4 With the notation and under the assumptions of Theorem 6.1 one has

$$
\|u(s)\|_{q} \geq C\left[T\left(u_{0}\right)-s\right]^{1 /(1-m)}
$$

for all $s \in\left[0, T\left(u_{0}\right)\right]$.

Proof. Just set $t=T\left(u_{0}\right)$ in $(6.2)$.

\section{Results on Cartan-Hadamard manifolds}

The following theorem, which contains information about what we may call a weakened form of the conservation of mass, also proves a lower bound on the extinction time (if any) in terms of local quantities related to the initial datum.

Theorem 7.1 Let $u(t, x) \in \mathrm{L}_{\text {loc }}^{1}(M)$ be a nonnegative solution of the fast diffusion equation on a Cartan-Hadamard manifold which Ricci curvature bounded below. Then for any $R>0, \alpha>1$ and $x_{0} \in M$ be such that $B_{\alpha R}=B_{\alpha R}\left(x_{0}\right) \subset M$, then

$$
\int_{B_{R}} u(t, x) \mathrm{d} x \leq 2^{1 /(1-m)}\left[\int_{B_{\alpha R}} u(s, x) \mathrm{d} x+\mathcal{M}_{R, \alpha}^{1 /(1-m)}|t-s|^{1 /(1-m)}\right]
$$


for any $t, s \geq 0$, where

$$
\mathcal{M}_{R, \alpha}=\frac{c_{0}}{(\alpha-1) R}\left(c_{1}+\frac{c_{0}}{(\alpha-1) R}\right) \operatorname{Vol}\left(B_{\alpha R} \backslash B_{R}\right)^{1-m}>0
$$

where the constants $c_{i}>0$ are independent of $u$ and $u_{0}$, and depend only on $m, d$ and on the lower bound for the Ricci curvature of $M$. If there exists a Finite Extinction Time $T\left(u_{0}\right)$, then it has a lower bound

$$
T\left(u_{0}\right) \geq c_{m, d}(\alpha-1) R\left(c_{1}+\frac{c_{0}}{(\alpha-1) R}\right)^{-1} \operatorname{Vol}\left(B_{\alpha R} \backslash B_{R}\right)^{-(1-m)}\left(\int_{B_{R}} u_{0} \mathrm{~d} x\right)^{1-m}
$$

\subsection{The Laplacian of the distance function}

We let $M$ be a Cartan-Hadamard manifold so that, given any fixed point $o \in M$, the exponential map is a diffeomorphism between $T_{o} M$ and $M$. The radial vector field on $M \backslash\{o\}$ is the unit vector field with the property that for any $x \in M \backslash\{o\}, \partial(x)$ is the unit tangent to the unique geodesic joining $o$ and $x$ and running from $o$.

Let $s \in(-a, a)$ and $\gamma_{s}: I \rightarrow M$ be a family of curves, $I$ being an interval in $\mathbb{R}$. The transversal vector field of $\{\gamma(s)\}$ along $\gamma_{0}$ is the vector field $W(t)$ along $\gamma_{0}$ defined by the fact that $W(t)$ is the tangent vector to the curve $s \mapsto \gamma_{s}(t)$ at $s=0$.

Let $D$ denote covariant differentiation with respect to the Levi-Civita connection. Let $\gamma_{0}$ be a geodesic parameterized by arclength, i.e. $|\dot{\gamma}|=1\left(|\cdot|\right.$ denotes the Riemannian norm in $\left.T_{X} M\right)$ and $D_{\dot{\gamma}} \dot{\gamma}=0$, and let $L(s)$ be the length of $\gamma_{s}$. The second variation formula states that, choosing $I=[0, b]$ and denoting $\gamma_{0}$ simply by $\gamma$ :

$$
L^{\prime \prime}(0)=\left.\left\langle D_{X} W, \dot{\gamma}\right\rangle\right|_{0} ^{b}+\int_{0}^{b} \mathrm{~d} t\left(\langle\dot{W}, \dot{W}\rangle-\langle R(W, \dot{\gamma}) W, \dot{\gamma}\rangle-\left(\langle W, \dot{\gamma}\rangle^{\prime}\right)^{2}\right)
$$

where $\dot{W}=D_{\dot{\gamma}} W,^{\prime}=\frac{\mathrm{d}}{\mathrm{d} t},\langle\cdot, \cdot\rangle$ denotes the Riemannian pairing and $R$ the curvature tensor.

The Hessian $D^{2} f$ of a scalar $f$ is the symmetric tensor field of type $(0,2)$ defined as

$$
D^{2} f(X, Y)=X(Y f)-\left(D_{X} Y\right) f \quad \forall X, y \in T M .
$$

The Laplace-Beltrami operator $\Delta$ then satisfies: $\Delta f=\operatorname{tr} D^{2} f$ so that $\Delta f(x)=\sum_{i} D^{2} f\left(e_{i}, e_{i}\right)$ where the sum is over an orthonormal basis $\left\{e_{i}\right\}$ at $x$.

Consider now the Riemannian distance $\varrho$ from the pole $o$.

Lemma 7.2 Let $M$ be a Cartan-Hadamard manifold. Then $D^{2} \varrho>0$ and, in particular, $\Delta \varrho>0$.

Proof. We adapt an argument of [13] Recall that the assumption that $M$ is a Cartan-Hadamard manifold amounts to assuming that $M$ is simply connected and that $\mathrm{Sec} \leq 0$, where Sec denotes sectional curvature.

Let $x \in M \backslash\{o\}$ with $\varrho(x)=b$ and $X \in T_{x} M, X \neq 0$ s.t. $\langle X, \partial\rangle=0$. There exists a curve $\zeta$ contained in the geodesic sphere or radius $b$ centered in $o$ such that $\dot{\zeta}(0)=X$, because $X$ is orthogonal to such a sphere. Let $\gamma_{s}:[0, b] \rightarrow M$ be the unique geodesic, parameterized by arclength, joining 
$o$ to $\zeta(s)$ and $W$ the corresponding transversal vector field. By construction $W(0)=0, W(b)=X$, $\left\langle W(t), \dot{\gamma}_{0}(t)\right\rangle=0$. It is clear that $L(s)=b$ for all $s$ so that

$$
\begin{aligned}
0 & =L^{\prime \prime}(0)=\left\langle D_{X} \dot{\zeta}, \partial(x)\right\rangle+\int_{0}^{b} \mathrm{~d} t(\langle\dot{W}, \dot{W}\rangle-\langle R(W, \dot{\gamma}) W, \dot{\gamma}\rangle) \\
& >\left\langle D_{X} \dot{\zeta}, \partial(x)\right\rangle
\end{aligned}
$$

where we have used the following facts. First, the boundary term at $t=0$ vanishes since $\langle W, \dot{\gamma}\rangle=0$ implies

$$
0=W(\langle W, \dot{\gamma}\rangle)=\left\langle D_{W} W, \dot{\gamma}\right\rangle+\left\langle W, D_{W} \dot{\gamma}\right\rangle
$$

so that $\left.\left\langle D_{W} W, \dot{\gamma}\right\rangle\right|_{0}=0$ since $W(0)=0$. Then we have used the fact that $W$ is not parallel so that $\dot{W}=D_{\dot{\gamma}} W \neq 0$ and hence $\langle\dot{W}, \dot{W}\rangle>0$ and finally the curvature condition.

We have then proved that $\left\langle D_{X} \dot{\zeta}, \partial(x)\right\rangle<0$. Notice now that $\partial(x)=\nabla \varrho(x)$ so that, by definition of Hessian and by the fact that $\varrho$ is constant on the geodesic sphere of radius $b$ :

$$
0 \geq\left\langle D_{X} \dot{\zeta}, \partial(x)\right\rangle=\left\langle D_{X} \dot{\zeta}, \nabla \varrho(x)\right\rangle=\left(D_{X} \dot{\zeta}\right) \varrho=-D^{2} \varrho(X, X) .
$$

Hence, for all nonzero vectors $X \in T_{x} M$ with $X \perp \partial(x)$, we have $D^{2} \varrho(X, X) \geq 0$. On the other hand

$$
D^{2} \varrho(\partial, \partial)=\partial(\partial \varrho)-\left(D_{\partial}\right) \partial \varrho=0
$$

since $D_{\partial} \partial=0$ and $\partial \varrho \equiv 1$. Finally, we use again the fact that $\partial \varrho \equiv 1$ and that $\partial(x)=\nabla \varrho$ to show that if $X \in T_{x} M$ is tangent to the geodesic sphere of radius $b$ one has:

$$
D^{2} \varrho(X, \partial)=-\left(D_{X} \partial\right) \varrho=-\left\langle D_{X} \partial, \nabla \varrho\right\rangle=-\left\langle D_{X} \partial, \partial\right\rangle=-\frac{1}{2} X\langle\partial, \partial\rangle=0 .
$$

\subsection{Weak Conservation of Mass}

In this section we prove in the Riemannian setting estimates on the behaviour of the local $\mathrm{L}^{1}$-norm of the solution. These estimates were first proved in Lemma 3.1 of [15], in the Euclidean setting. We shall use them to provide lower bounds on the FET, as first noticed in [6].

Notice that, since these estimates have local nature, they hold true both for the Cauchy problem on the whole manifold $M$, but also for any mixed Dirichlet-Cauchy problem on a domain $N \subset M$ with or without finite measure.

Proposition 7.3 Let $M$ be a Cartan-Hadamard manifold with Ricci curvature bounded below. Let $u(t, x) \geq v(t, x) \in \mathrm{L}_{l o c}^{1}(M)$ be such that

$$
\begin{aligned}
& u, v \in C\left([0,+\infty) ; \mathrm{L}_{l o c}^{1}(M)\right) \\
& u_{t}=\Delta\left(u^{m}\right), \quad v_{t}=\Delta\left(v^{m}\right), \quad \text { in } \mathcal{D}^{\prime}((0,+\infty) \times M)
\end{aligned}
$$

where $a^{m}=|a|^{m-1} a$. Let $R>0, \alpha>1$ and $x_{0} \in M$ be such that $B_{\alpha R}=B_{\alpha R}\left(x_{0}\right) \subset M$, then

$$
\left[\int_{B_{R}}[u(t, x)-v(t, x)] \mathrm{d} x\right]^{1-m} \leq\left[\int_{B_{\alpha R}}[u(s, x)-v(s, x)] \mathrm{d} x\right]^{1-m}+\mathcal{M}_{R, \alpha}|t-s|,
$$


for any $t, s \geq 0$, where

$$
\mathcal{M}_{R, \alpha}=\frac{c_{0}}{(1-\alpha) R}\left(c_{1}+\frac{c_{0}}{(\alpha-1) R}\right) \operatorname{Vol}\left(B_{\alpha R} \backslash B_{R}\right)^{1-m}>0
$$

where the constants $c_{i}>0$ are independent of $u$ and $u_{0}$, and depend only on $m, d$ and on the lower bound for the Ricci curvature of $M$.

Proof. First we apply (7.3) to $u$ and $v$, to get

(7.6) $-\int_{0}^{\infty} \int_{M} \vartheta^{\prime}(t) \psi(x)[u(t, x)-v(t, x)] \mathrm{d} x \mathrm{~d} t=\int_{0}^{\infty} \int_{M} \vartheta(t) \Delta(\psi(x))\left[u^{m}(t, x)-v^{m}(t, x)\right] \mathrm{d} x \mathrm{~d} t$ where $\vartheta \in C_{c}^{\infty}(0,+\infty)$ and $\psi \in C_{c}^{\infty}(M)$. This imply

$$
\frac{\mathrm{d}}{\mathrm{d} t} \int_{M} \psi(x)[u(t, x)-v(t, x)] \mathrm{d} x=\int_{M} \Delta(\psi(x))\left[u^{m}(t, x)-v^{m}(t, x)\right] \mathrm{d} x
$$

in $\mathcal{D}^{\prime}(0,+\infty)$ and therefore in $\mathrm{L}_{l o c}^{1}(0,+\infty)$ as well.

The numerical inequality

$$
a^{m}-b^{m} \leq 2^{1-m}(a-b)^{m}
$$

valid if $a \geq b \in \mathbb{R}, a^{m}=|a|^{m-1} a$ and $0<m<1$; this gives together with (7.7)

$$
\left|\frac{\mathrm{d}}{\mathrm{d} t} \int_{M} \psi(x)[u(t, x)-v(t, x)] \mathrm{d} x\right| \leq 2^{1-m} \int_{M}|\Delta(\psi(x))|[u(t, x)-v(t, x)]^{m} \mathrm{~d} x
$$

Setting now $w=u-v \geq 0$, by Hölder inequality we get the differential inequality

$$
\left|\frac{\mathrm{d}}{\mathrm{d} t} \int_{M} \psi(x) w(t, x) \mathrm{d} x\right| \leq C(\psi)\left[\int_{M} \psi(x) w(t, x) \mathrm{d} x\right]^{m}
$$

where

$$
C(\psi)=2^{1-m}\left[\int_{M}|\Delta(\psi(x))|^{1 /(1-m)} \psi(x)^{-m /(1-m)} \mathrm{d} x\right]^{1-m} .
$$

Integrating the differential inequality (7.9) will give

$$
\left[\int_{M} \psi(x) w(t, x) \mathrm{d} x\right]^{1-m} \leq\left[\int_{M} \psi(x) w(s, x) \mathrm{d} x\right]^{1-m}+(1-m) C(\psi)|t-s|
$$

for any $s, t \geq 0$. This will immediately imply the statement, once we prove that $C(\psi)=\mathcal{M}(R, \alpha)<$ $+\infty$.

To this end we consider a function $\psi=\varphi^{b} \in C_{c}^{\infty}(M)$, with

$$
0 \leq \varphi \leq 1, \quad \varphi \equiv 1 \text { in } B_{R}, \quad \varphi \equiv 0 \text { outside } B_{\alpha R}
$$

with $\alpha>1$. Moreover we will assume that $\varphi$ is "radial" and

$$
\varphi(x)=\bar{\varphi}(\varrho(x) / R)
$$


where $\bar{\varphi}: \mathbb{R} \rightarrow \mathbb{R}$ is a $C_{c}^{\infty}(\mathbb{R})$ function such that:

$$
0 \leq \bar{\varphi}(s) \leq 1, \quad \bar{\varphi}(s) \equiv 1, \text { for } 0 \leq s \leq 1, \quad \bar{\varphi} \equiv 0, \text { for } s \geq \alpha
$$

where $\alpha>1$ and $\varrho(x)$ is the Riemannian distance from a fixed point. We then have

$$
\begin{gathered}
|\Delta(\psi(x))|^{1 /(1-m)} \psi(x)^{-m /(1-m)}=\left.\varphi(x)^{-b m /(1-m)}\left|b(b-1) \varphi^{b-2}\right| \nabla \varphi\right|^{2}+\left.b \varphi^{b-1} \Delta \varphi\right|^{1 /(1-m)} \\
\leq\left.[b(b-1)]^{1 /(1-m)} \varphi^{[(b-2)-b m] /(1-m)}|| \nabla \varphi\right|^{2}+|\Delta \varphi|^{1 /(1-m)}
\end{gathered}
$$

the last inequality follow from the fact that we are considering a radial function $0 \leq \varphi(x)=$ $\bar{\varphi}(\varrho(x) / R) \leq 1$, with $b>2 /(1-m)$. We know by Lemma 7.2 that $\Delta \varrho>0$ in our setting.

We next recall that Calabi's inequalities, sometimes known also as the Laplacian comparison Theorem (cf. [13]) states that, if Ric $\geq-(d-1) a^{2}$

$$
\Delta \varrho \leq(d-1) a \operatorname{coth}(a \varrho)
$$

where the r.h.s. is in fact the Laplacian of $\varrho$ in a space of fixed, constant negative curvature. Therefore

$$
\Delta \varrho \leq c_{1}+\frac{c_{2}}{\varrho}
$$

where of course in the Euclidean case $c_{1} \equiv 0$.

We have then shown that $|\Delta \varrho| \leq c_{1}+c_{2} / \varrho$. Then we compute

$$
\begin{aligned}
|\nabla \varphi(x)|^{2} & =R^{-2}\left|\bar{\varphi}^{\prime}(\varrho(x) / R)\right|^{2}|\nabla \varrho|^{2} \leq R^{-2}\left|\bar{\varphi}^{\prime}(\varrho(x) / R)\right|^{2} \leq c_{\alpha} R^{-2} \\
|\Delta \varphi(x)| & =\left.\left|R^{-2} \bar{\varphi}^{\prime \prime}(\varrho(x) / R)\right| \nabla(\varrho(x))\right|^{2}+R^{-1} \bar{\varphi}^{\prime}(\varrho(x) / R) \Delta \varrho(x) \mid \\
& \leq R^{-1}\left[\left|R^{-1} \bar{\varphi}^{\prime \prime}(\varrho(x) / R)\right||\nabla(\varrho(x))|^{2}+\left|\bar{\varphi}^{\prime}(\varrho(x) / R)\right||\Delta \varrho(x)|\right] \\
& \leq R^{-1}\left(\left|R^{-1} \bar{\varphi}^{\prime \prime}(\varrho(x) / R)\right|+\left|\bar{\varphi}^{\prime}(\varrho(x) / R)\right|\left(c_{1}+\frac{c_{2}}{\varrho}\right)\right) \\
& \leq c_{\alpha} \frac{1}{R}\left(c_{1}+\frac{c_{\alpha}}{R}\right)
\end{aligned}
$$

where in the last step we used the fact that $\Delta \varphi$ is supported in $D_{R, \alpha}=B_{\alpha R} \backslash B_{R}$ and that the smooth function $\bar{\varphi}$ has bounded derivatives in $D_{R, \alpha}$

$$
\bar{\varphi}^{\prime \prime}(\varrho(x) / R)|+| \bar{\varphi}^{\prime}(\varrho(x) / R) \mid \leq \frac{c_{0}}{(\alpha-1)}=c_{\alpha}
$$

we just remark that this last estimate depend on an explicit choice of the test function $\bar{\varphi}$. An integration over $D_{R, \alpha}$ gives:

$$
\begin{aligned}
C(\psi) & =2^{1-m}\left[\int_{D_{R, \alpha}}|\Delta(\psi(x))|^{1 /(1-m)} \psi(x)^{-m /(1-m)} \mathrm{d} x\right]^{1-m} \\
& \leq \frac{c_{0}}{(\alpha-1) R}\left(c_{1}+\frac{c_{0}}{(\alpha-1) R}\right) \operatorname{Vol}\left(D_{R, \alpha}\right)^{1-m}
\end{aligned}
$$

This concludes the proof.

Remark 7.4 The proof shows that in the Euclidean case the constant $c_{1}$ vanishes.

Clearly, Theorem 7.1 is a consequence of the above result. 


\subsection{Volume growth}

CASE 1) If the volume growth of the balls of $M$ is sub-exponential

$$
\operatorname{Vol}\left(B_{R}\right) \leq C(d, M) R^{\sigma}, \quad \sigma>0
$$

or

$$
\mathcal{M}=C_{m, d}[(\alpha-1) R]^{-2+\sigma(1-m)}
$$

The case of $M=\mathbb{R}^{d}$ is contained in this one, since we have $\sigma=d$. Letting $\alpha=2$ we recover Lemma 3.1 of $[15]$. In the $\mathbb{R}^{d}$-case the lower bound on extinction time becomes:

$$
T\left(u_{0}\right) \geq C_{m, d} R^{2-d(1-m)}\left(\int_{B_{R}} u_{0} \mathrm{~d} x\right)^{1-m}
$$

The quantity $2-d(1-m)$ is positive if $m>m_{c}=(d-2) / d$ and negative if $m<m_{c}$. Letting then $R \rightarrow \infty$ in the case $m>m_{c}$ gives $T=+\infty$, and this shows that if the initial datum is nonzero in some sets of positive measure, then the solution does not extinguish in finite time, while this is not always true in the case $m<m_{c}$.

CASE 2) If the volume growth of the balls of $M$ is exponential

$$
\operatorname{Vol}\left(B_{R}\right) \sim C(d, M) \mathrm{e}^{\sigma R}, \quad \sigma>0, R \rightarrow+\infty
$$

then the lower bound on the FET becomes

$$
T\left(u_{0}\right) \geq C_{m, d} R^{2} \mathrm{e}^{-\sigma R}\left(\int_{B_{R}} u_{0} \mathrm{~d} x\right)^{1-m} .
$$

in particular for all $0<m<1$ one cannot a priori conclude that there is a solution which is not vanishing in finite time. This is consistent with the results of the previous Section.

Remark (2) still holds when the volume growth of the balls is faster than exponential.

\section{Summary of the results}

(I) The supercritical case: $m>m_{c}$. In this zone there is a different behaviour depending on the curvature and on the volume.

Euclidean Space $\mathbb{R}^{d}$. There is not extinction in finite time, for data in any $\mathrm{L}^{p}\left(\mathbb{R}^{d}\right)$, with $p \geq 1$, moreover there holds the smoothing effect, from any $\mathrm{L}^{p}\left(\mathbb{R}^{d}\right)$, with $p \geq 1$ to $\mathrm{L}^{\infty}\left(\mathbb{R}^{d}\right)$. There holds the conservation of total mass. In the cases of bounded domains when we consider the mixed CauchyDirichlet problem, there is moreover the extinction in finite time, due to the fact that the Poincaré inequality holds. There is no conservation of mass.

Negative Curvature. In the case of nonparabolic manifolds with infinite volume and negative curvature, there is extinction in finite time, for any data in any $\mathrm{L}^{p}\left(\mathbb{R}^{d}\right)$, with $p \geq 1$, due to the Poincaré inequality, or spectral gap, and there the smoothing effect holds and it is equivalent to Sobolev inequalities. The same holds in any subdomains therein when we consider the mixed Cauchy-Dirichlet problem. In all situations the conservation of total mass does not hold. 


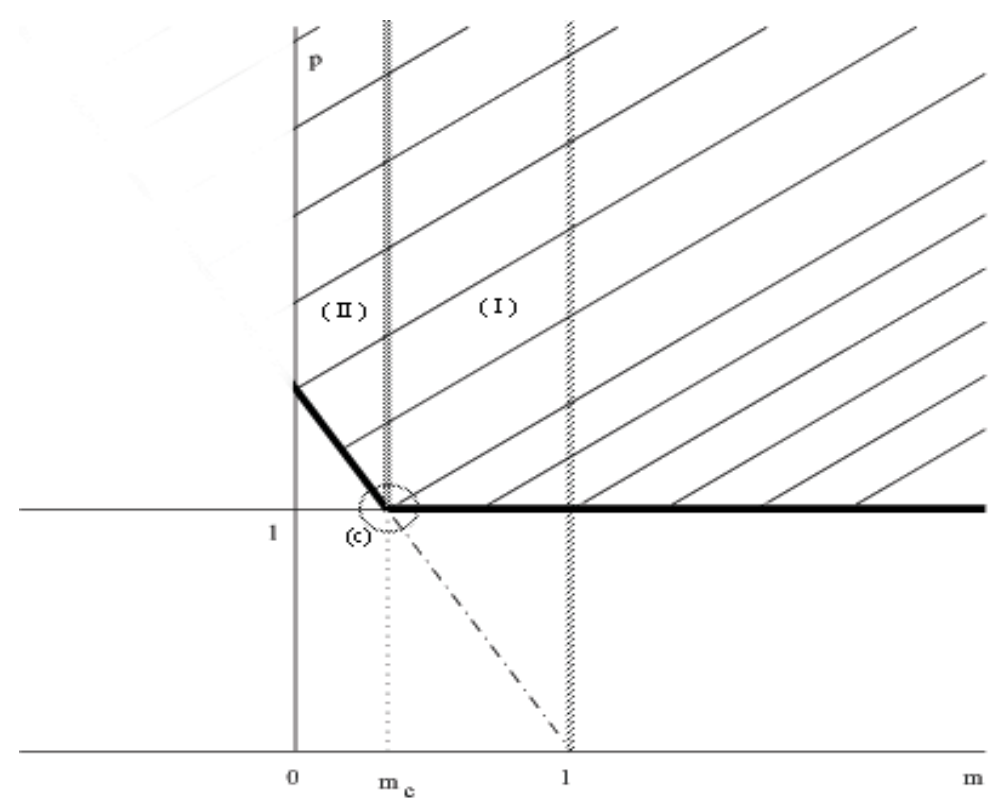

(II) The subcritical case: $0<m<m_{c}$. In this zone the behaviour seems not to depend on the curvature and on the volume. Indeed, the mass is not conserved in any case, and the solution extinguishes in finite time in any situation: in the case of nonpositive curvature, both with infinite volume, and also for finite volume with additional Dirichlet conditions. The Smoothing Effect holds for any data in any $\mathrm{L}^{p}\left(\mathbb{R}^{d}\right)$, with $p>p_{c}=d(1-m) / 2$.

The point $(c)=\left(m_{c}, 1\right)$ is a critical case in which solutions do not extinguish in finite time neither in $\mathbb{R}^{d}$ nor in bounded domains therein.

Acknowledgment. JLV was partially supported by Spanish Project MTM2005-08760-C02-01 and ESF Programme "Global and geometric aspects of nonlinear partial differential equations". MB was supported by the same program to visit the Universidad Autónoma de Madrid. 


\section{References}

[1] D. Bakry, T. Coulhon, M. Ledoux, L. Saloff-Coste, Sobolev inequalities in disguise, Indiana Univ. Math. J. 44 (1995), 1033-1074.

[2] Ph. Bénilan, M. G. Crandall, The continuous dependence on $\varphi$ of solutions of $u_{t}-\Delta \varphi(u)=0$, Indiana Univ. Math. J. 30 (1981), 161-177.

[3] M. Bonforte, F. Cipriani, G. Grillo, Ultracontractivity and approach to equilibrium for supercritical parabolic equations on riemannian manifolds, Adv. Differential Equations 8 (2003), 843-872.

[4] M. Bonforte, G. Grillo, Asymptotics of the porous media equation via Sobolev inequalities, J. Funct. Anal. 225, (2005), 33-62

[5] M. Bonforte, G. Grillo, Hyper and ultracontractive bounds for doubly nonlinear evolution equations, Rev. Mat. Iberoamericana 22 (2006), 111-129.

[6] M. Bonforte, J. L. Vazquez, Global Positivity Estimates and Harnack Inequalities for the Fast Diffusion Equation, Preprint (January 2006).

[7] M. Bonforte, J. L. Vazquez, Fine Asymptotics near Extinction and Elliptic Harnack Inequalities for the Fast Diffusion Equation, Preprint (March 2006).

[8] H. Brezis, Monotonicity methods in Hilbert spaces and some applications to nonlinear partial differential equations, Proc. Symp. Nonlinear Funct. Anal., Madison, Acad. Press (1971), 101156.

[9] G. Carron, Inégalités isopérimétriques de Faber-Krahn et conséquences, Publ. Inst. Fourier 220 (1992).

[10] F. Cipriani, G. Grillo, Uniform bounds for solutions to quasilinear parabolic equations, J. Diff. Equations. 177 (2001), 209-234.

[11] E. B. Davies, Heat Kernels and Spectral Theory, Cambridge University Press, 1989.

[12] E. DiBenedetto, Degenerate Parabolic Equations, Springer Verlag, New York/Berlin, 1993.

[13] R. E. Green, H. Wu, Function theory on manifolds which possess a Pole, Lecture Notes in Mathematics 699, 1979.

[14] E. Hebey, Nonlinear Analysis on Manifolds: Sobolev Spaces and Inequalities, American Mathematical Society, 2000.

[15] M. A. Herrero, M. Pierre, The Cauchy Problem for $u_{t}=\Delta u^{m}$ when $0<m<1$, Trans. Amer. Math. Soc. 291 n.1 (1985), 145-158.

[16] O. A. Ladyzhenskaya, V. A. Solonnikov \& N. N. Ural'tseva, Linear and Quasilinear Equations of Parabolic Type, Transl. Math. Monographs, 23, Amer. Math. Soc, Providence, RI, 1968.

[17] J. L. Vázquez, The Porous Medium, Mathematical Theory, (2006) Oxford University Press, to appear.

[18] J. L. Vázquez, Smoothing and Decay Estimates for Nonlinear Diffusion Equations. Equations of Porous Medium Type, (2006) Oxford Univ. Press, to appear. 